\title{
Significance of an Epidermal Growth Factor Receptor Mutation in Cerebrospinal Fluid for Carcinomatous Meningitis
}

\author{
Nobuhiro Kanaji ${ }^{1}$, Shuji Bandoh ${ }^{1}$, Norihiro Nagamura ${ }^{1}$, \\ Yoshio Kushida ${ }^{2}$, Reiji $\mathrm{Haba}^{2}$ and Toshihiko Ishida ${ }^{1}$
}

\begin{abstract}
We report a case of epidermal growth factor receptor (EGFR) inhibitor-sensitive lung adenocarcinoma with carcinomatous meningitis who showed a good response to gefitinib, an oral tyrosine kinase inhibitor of EGFR. This good response to gefitinib treatment was attributed to evidence of an EGFR mutation, L858R in exon 21, which was detected in a small amount of cerebrospinal fluid (CSF) before the positive CSF cytology. Patients with carcinomatous meningitis often have a poor performance status, and therefore diagnostic approaches and therapeutic methods are also often limited. Detection of EGFR mutations may be a useful method for non-small cell lung cancer diagnosis, and also facilitate determination of appropriate therapeutic protocols.
\end{abstract}

Key words: non-small cell lung cancer, epidermal growth factor receptor, carcinomatous meningitis, cerebrospinal fluid, gefitinib, mutation

(DOI: 10.2169/internalmedicine.46.0335)

\section{Introduction}

Carcinomatous meningitis is a clinically important neurological complication of systemic cancer, and often leads to a poor performance status. The prognosis for patients with carcinomatous meningitis is very poor, with a median survival of only 4 weeks (1).

Gefitinib (Iressa ${ }^{\mathrm{TM}}$; AstraZeneca, Wilmington, DE) is an oral tyrosine kinase inhibitor of epidermal growth factor receptor (EGFR), and large clinical trials have demonstrated its anticancer activity in patients with non-small cell lung cancer (NSCLC) $(2,3)$. It has also been reported that mutations of the EGFR gene can predict prolonged survival after gefitinib treatment in patients with $\operatorname{NSCLC}(4,5)$. Several case reports have indicated that gefitinib may also be effective against carcinomatous meningitis (6-8).

We report a NSCLC patient with carcinomatous meningitis, in whom an EGFR mutation was detected that brought about a good clinical response to gefitinib treatment. To the best of our knowledge, this is the first report demonstrating evidence of an EGFR mutation in cerebrospinal fluid (CSF). Furthermore, this case suggests that an EGFR mutation can be detected in a small amount of CSF, despite negative cytology.

\section{Case Report}

A 72-year-old man was admitted to our hospital in January 2007 for further evaluation of disturbance of consciousness that had gradually progressed for 1 month. His consciousness level was in the twilight state, and he was completely unable to express his intentions. There was no fever elevation. He had smoked about 20 cigarettes for 50 years.

Head computed tomography (CT) revealed dilatation of the cerebral ventricles, periventricular low density and enhancement on the brain surface (Fig. 1A), suggesting meningitis, especially carcinomatous meningitis, together with his clinical course. A chest X-ray showed a nodule in the right middle lung field, and CT revealed a $3-\mathrm{cm}$ tumor with ir-

\footnotetext{
${ }^{1}$ Department of Internal Medicine, Division of Endocrinology and Metabolism, Hematology, Rheumatology and Respiratory Medicine, Faculty of Medicine, Kagawa University, Kagawa and ${ }^{2}$ Department of Diagnostic Pathology, Faculty of Medicine, Kagawa University, Kagawa Received for publication May 23, 2007; Accepted for publication July 11, 2007 Correspondence to Dr. Nobuhiro Kanaji, kanaji@med.kagawa-u.ac.jp
} 


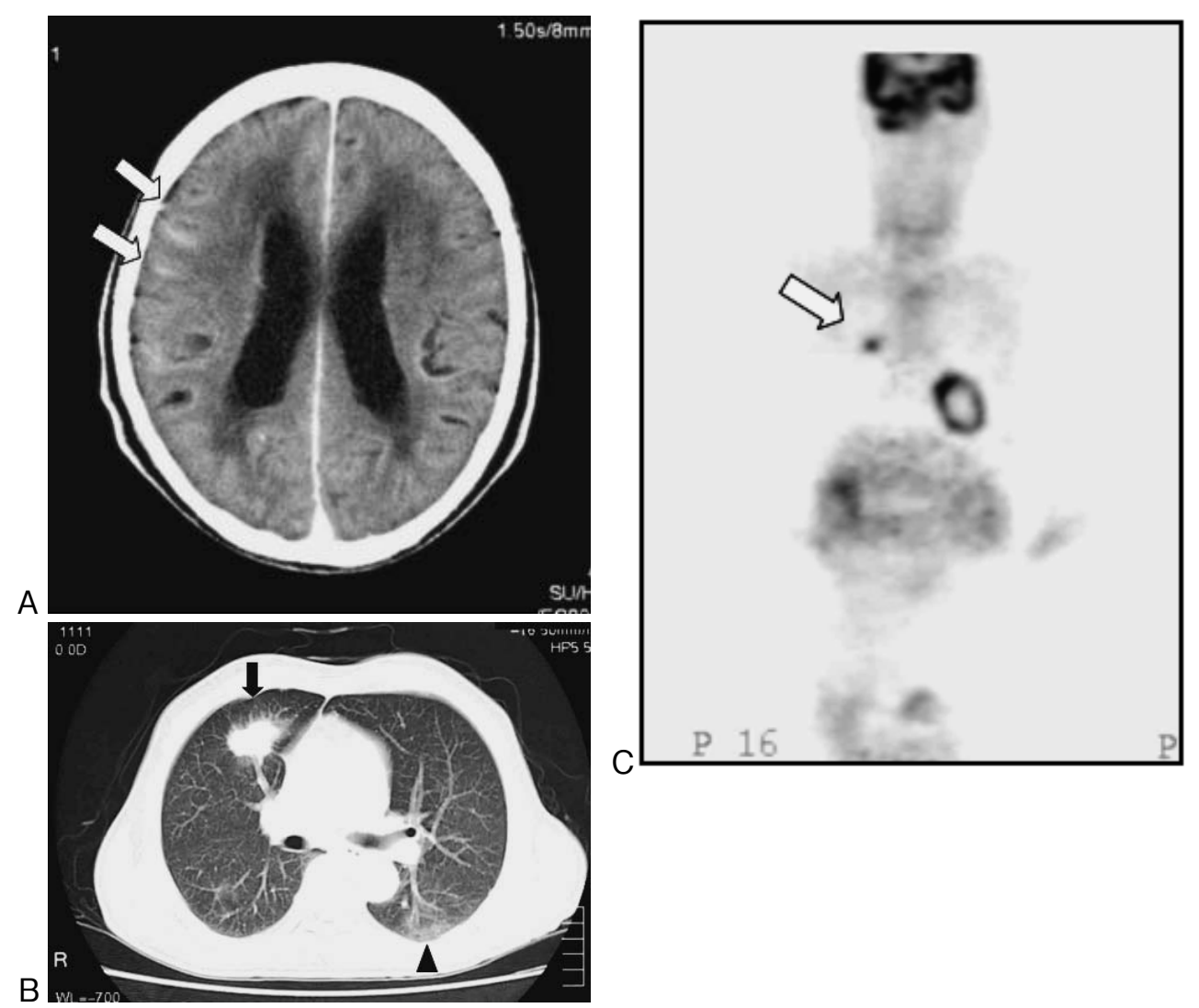

Figure 1. (A) Head CT shows enhancement of the brain surface, indicating carcinomatous meningitis (arrow). (B) Chest CT shows primary lung cancer in the right upper lobe (arrow) and aspiration pneumonia in the left $\mathrm{S}^{6}$ area (arrowhead). (C) There is no abnormal uptake of F18-fluorodeoxyglucose except for the primary lesion (arrow) on positron emission tomography.

regular edges in the right upper lobe (Fig. 1B), suggesting lung adenocarcinoma. Aspiration pneumonia due to his deteriorated consciousness was also recognized (Fig. 1B). Abnormal F18-fluorodeoxyglucose uptake (SUV 6.7) into the tumor was seen on positron emission tomography scanning, and there was no other abnormal uptake into the lesion (Fig. 1C). The serum carcinoembryonic antigen (CEA) level was $1.5 \mathrm{ng} / \mathrm{ml}$ and within the normal range. Although primary lung cancer with carcinomatous meningitis was highly suspected, a bronchoscopic approach could not be performed because of his twilight state. Lumbar punctures were performed to diagnose the cause of the disturbance of consciousness. The cerebrospinal pressure was $18 \mathrm{mmH}_{2} \mathrm{O}$ and the cell number in the CSF was slightly increased to $21 \mu$ / $\mathrm{ml}$. The CEA level in the CSF was $6.1 \mathrm{ng} / \mathrm{ml}$, which was 4fold higher than the serum CEA level. Although two lumbar punctures were performed and each 3-ml CSF sample was examined for cytodiagnosis, two clinical pathologists did not diagnose a cancerous lesion. No microorganisms, including Mycobacterium tuberculosis, were identified in the CSF. In a third lumbar puncture, carcinomatous meningitis was established by positive cytology for malignant cells consistent with metastatic adenocarcinoma (Fig. 2). He was diagnosed as lung cancer with carcinomatous meningitis (cT1NOM1, stage IV).

We obtained informed consent for genetic analysis from

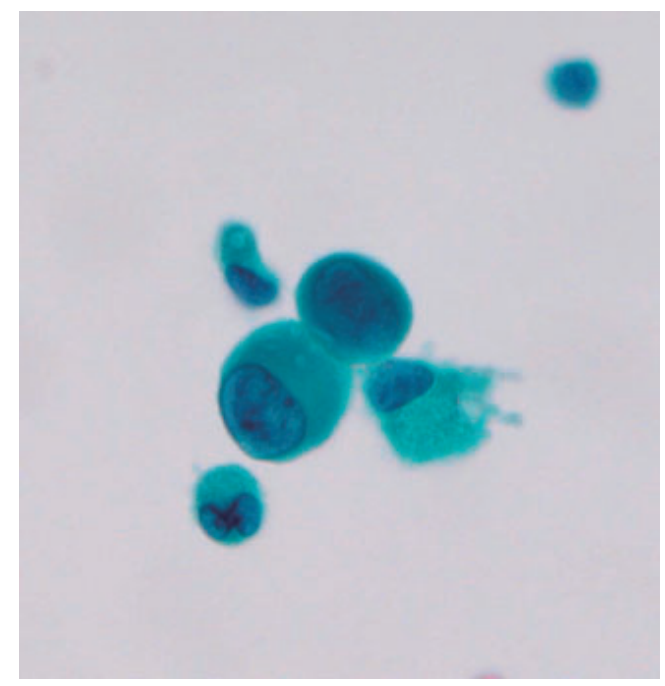

Figure 2. Cytologic examination of the CSF obtained from the third lumbar puncture is consistent with adenocarcinoma (Papanicolaou stain, $\times 400)$.

the patient's family and analyzed the EGFR status in the CSF obtained from the first lumbar puncture. Total ribonucleic acid (RNA) was extracted from the cells in $0.5 \mathrm{ml}$ of the CSF sample using a QIAamp RNA Blood Mini Kit (Qiagen, Hilden, Germany), and $40 \mu \mathrm{l}$ of complementary de- 


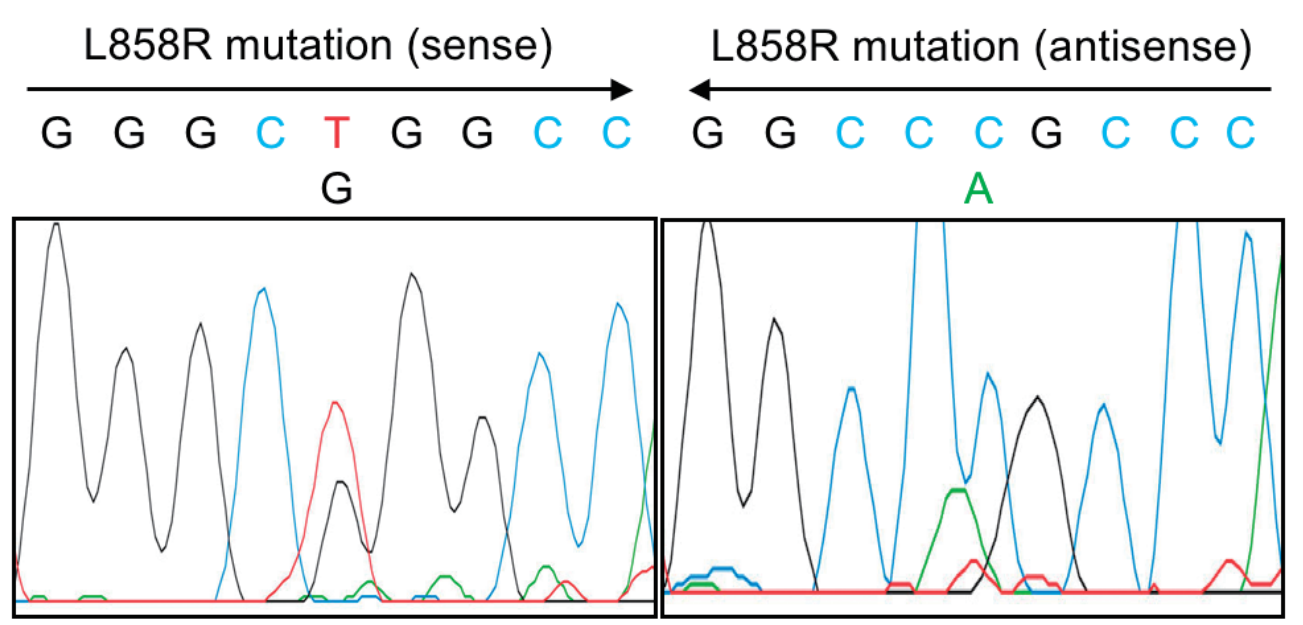

Figure 3. Sequencing of EGFR from the patient's CSF sample confirms the L858R mutation.

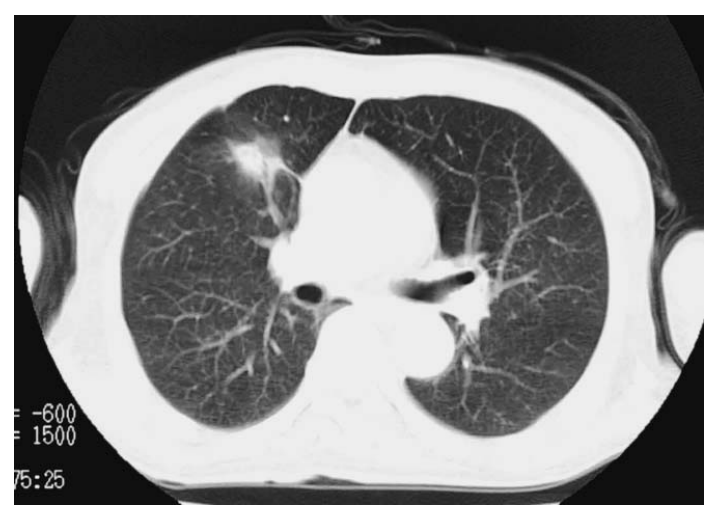

Figure 4. The primary lesion regressed significantly 2 months after initiating gefitinib therapy.

oxyribonucleic acid (cDNA) was synthesized using an oligo dT primer and SuperScript II RNaseH-Reverse Transcriptase (Invitrogen, Carlsbad, CA). The sequences of the genespecific primers for the reverse transcription-polymerase chain reaction (RT-PCR) were as follows: F1, 5'-AGCTTGTGGAGCCTCTTACACC-3'; R1,

5'-TAAAATTGATTCCAATGCCATCC-3'; F2,

5'-TCCTCGATGAAGCCTACGTGA-3'; R2,

5'-TGCCTCCTTCTGCATGGTATT-3'. The first PCR was performed using primers $\mathrm{F} 1$ and $\mathrm{R} 1$ followed by the second PCR using primers F2 and R2. The nested PCR was performed using Ready-To-Go PCR beads (Amersham Biosciences UK Ltd., Buckinghamshire, UK). Briefly, $1 \mu \mathrm{l}$ of each primer $(10 \mu \mathrm{M}), 1 \mu \mathrm{l}$ of cDNA and $22 \mu \mathrm{l}$ of distilled water were added to the PCR beads. The PCR amplification comprised of 30 cycles of denaturation at $94^{\circ} \mathrm{C}$ for $30 \mathrm{sec}-$ onds, annealing at $48^{\circ} \mathrm{C}\left(55^{\circ} \mathrm{C}\right.$ in the second PCR) for 30 seconds and extension at $72^{\circ} \mathrm{C}$ for 1 minute. The second PCR product was a 348-bp fragment. The EGFR mutation in the patient's CSF sample was examined using PCR-based direct sequencing. The DNA bands were isolated from the gel, purified and sequenced using the PRISM dye terminator cycle sequencing method and an ABI PRISM 310 Genetic Analyzer version 3.4.5 (Applied Biosystems, Foster City,
CA). As a result, the L858R mutation in exon 21 was confirmed to be present in the patient's CSF sample (Fig. 3). The other EGFR mutations were not detected (data not shown).

Since his Eastern Cooperative Oncology Group performance status was 4, conventional intravenous chemotherapy was considered to be risky. On the other hand, the presence of EGFR mutation was evident. Although gefitinib therapy could not be allowed for the first therapy, he was treated with gefitinib (250 mg/day) with his family's agreement. After 2 weeks, his consciousness level had improved significantly. After 2 months, his consciousness became alert, and the primary lesion was markedly regressed (Fig. 4). The clinical effectiveness to gefitinib treatment was a partial response.

\section{Discussion}

The present case suggests that gefitinib may be a powerful therapeutic option for carcinomatous meningitis in NSCLC patients. To the best of our knowledge, this is the first report to show a positive association between the evidence of an EGFR mutation in the CSF and a great benefit of gefitinib treatment for carcinomatous meningitis.

Carcinomatous meningitis is often found in patients with metastatic malignancies, and can also be the initial manifestation of an underlying malignancy (9). Although the diagnosis can generally be made by cytologic examination of the CSF, it has been reported that the initial cytologic examination is only diagnostic in approximately $50 \%$ of the cases (10). In the present case, the EGFR mutation was identified in the first CSF, despite negative CSF cytology. Among lung cancers, the most frequently involved histological types are adenocarcinoma and small cell lung cancer (1), and EGFR mutations are seen in the majority of adenocarcinomas (4). Since the consciousness level and performance status of patients with carcinomatous meningitis are frequently deteriorated, as in the present case, bronchoscopy often cannot be performed. Therefore, the detection of EGFR mutations in 
the CSF may help a diagnosis of lung cancer with carcinomatous meningitis. Although a recent study reported that EGFR mutations were also detected in esophageal and pancreatic adenocarcinomas (11), the present case showed typical radiological findings of a well-differentiated lung adenocarcinoma, suggesting that the metastatic carcinomatous meningitis was caused by the lung cancer.

The detection of EGFR mutations is not limited to a diagnosis of lung cancer, but can also lead to the use of optimal therapeutic methods, such as the choice of an EGFR inhibitor. The treatments for carcinomatous meningitis include radiotherapy, systemic chemotherapy and intrathecal chemotherapy with methotrexate, thiotepa and cytarabine (12). However, there is no established treatment. EGFR inhibitor treatment was reported to be effective in 24 of 29 patients with EGFR mutations, compared with 2 of 21 patients without mutations (4). The present case suggests that the presence of an EGFR mutation in the CSF may predict a good response to EGFR inhibitor administration in patients with carcinomatous meningitis. There have been several other case reports showing the efficacy of gefitinib for carcinomatous meningitis (6-8) as well as brain metastasis (13-15). EGFR inhibitors may become a new therapeutic option for carcinomatous meningitis. On the other hand, gefitinib may cause a serious adverse effect such as interstitial lung disease. It has been reported that gefitinib-induced interstitial lung disease was significantly associated with man sex, a history of smoking, and the coincidence of interstitial pneumonia (16). Another multivariate analysis revealed that preexisting pulmonary fibrosis, poor performance status, and prior thoracic irradiation were independent risk factors for interstitial lung disease (17). In the present case, smoking history, sex, and poor performance status were risk factors of gefitinib-induced interstitial lung disease. The analysis of EGFR mutation may be very important to determine whether treatment for EGFR inhibitor is proper or not.

Another suggestive finding in the present case was the elevated CEA level in the CSF. Several reports have demonstrated increased CEA levels in the CSF of most patients with carcinomatous meningitis arising from several kinds of cancer $(18,19)$. There are two possible mechanisms to explain the elevation of CEA in the CSF: (i) CEA is locally produced by cancer cells; and (ii) CEA is passively transferred by the serum, due to elevated serum CEA levels and/ or blood-CSF-barrier disturbance (18). In the present case, the CEA level in the CSF was 4-fold higher than the serum CEA level. This finding suggests that cancer cells in the CSF may produce CEA. In other words, cancer cells are present in the CSF. Although the increased CEA level in the CSF may suggest carcinomatous meningitis, it is not specific for lung cancer, and does not provide any information regarding the therapeutic strategy, including the prediction of effectiveness for EGFR inhibitor administration.

In conclusion, the evidence of an EGFR mutation in the CSF may help a diagnosis of lung cancer with carcinomatous meningitis and predict a good response to EGFR inhibitor treatment. In patients with a poor performance status, who are suspected of having lung cancer with carcinomatous meningitis, analysis of EGFR mutations in the CSF may be a very useful method to resolve two problems at one time.

\section{References}

1. Hammerer V, Pauli G, Quoix E. Retrospective study of a series of 26 carcinomatous meningitis secondary to lung cancer. Bull Cancer 92: 989-994, 2005.

2. Fukuoka M, Yano S, Giaccone G, et al. Multi-institutional randomized phase II trial of gefitinib for previously treated patients with advanced non-small-cell lung cancer (The IDEAL 1 Trial). J Clin Oncol 21: 2237-2246, 2003.

3. Cella D, Herbst RS, Lynch TJ, et al. Clinically meaningful improvement in symptoms and quality of life for patients with nonsmall-cell lung cancer receiving gefitinib in a randomized controlled trial. J Clin Oncol 23: 2946-2954, 2005.

4. Mitsudomi T, Kosaka T, Endoh H, et al. Mutations of the epidermal growth factor receptor gene predict prolonged survival after gefitinib treatment in patients with non-small-cell lung cancer with postoperative recurrence. J Clin Oncol 23: 2513-2520, 2005.

5. Han SW, Kim TY, Lee KH, et al. Clinical predictors versus epidermal growth factor receptor mutation in gefitinib-treated nonsmall-cell lung cancer patients. Lung Cancer 54: 201-207, 2006.

6. Kim MK, Lee KH, Lee JK, Choi JH, Hyun MS. Gefitinib is also active for carcinomatous meningitis in NSCLC. Lung Cancer 50: 265-269, 2005.

7. Sakai M, Ishikawa $\mathrm{S}$, Ito $\mathrm{H}$, et al. Carcinomatous meningitis from non-small-cell lung cancer responding to gefitinib. Int J Clin Oncol 11: 243-245, 2006.

8. Hashimoto N, Imaizumi K, Honda T, et al. Successful re-treatment with gefitinib for carcinomatous meningitis as disease recurrence of non-small-cell lung cancer. Lung Cancer 53: 387-390, 2006.

9. Janne PA, Janicek MJ, Brown F. Meningeal carcinomatosis in lung cancer. Case 2. Carcinomatous meningitis. J Clin Oncol 18: 2927-2929, 2000.

10. Grossman SA, Krabak MJ. Leptomeningeal carcinomatosis. Cancer Treat Rev 25: 103-119, 1999.

11. Kwak EL, Jankowski J, Thayer SP, et al. Epidermal growth factor receptor kinase domain mutations in esophageal and pancreatic adenocarcinomas. Clin Cancer Res 12: 4283-4287, 2006.

12. Grossman SA, Finkelstein DM, Ruckdeschel JC, Trump DL, Moynihan T, Ettinger DS. Randomized prospective comparison of intraventricular methotrexate and thiotepa in patients with previously untreated neoplastic meningitis. Eastern Cooperative Oncology Group. J Clin Oncol 11: 561-569, 1993.

13. Chiu CH, Tsai CM, Chen YM, Chiang SC, Liou JL, Perng RP. Gefitinib is active in patients with brain metastases from nonsmall cell lung cancer and response is related to skin toxicity. Lung Cancer 47: 129-138, 2005.

14. Poon AN, Ho SS, Yeo W, Mok TS. Brain metastasis responding to gefitinib alone. Oncology 67: 174-178, 2004.

15. Namba Y, Kijima T, Yokota $S$, et al. Gefitinib in patients with brain metastases from non-small-cell lung cancer: review of 15 clinical cases. Clin Lung Cancer 6: 123-128, 2004.

16. Ando M, Okamoto I, Yamamoto N, et al. Predictive factors for interstitial lung disease, antitumor response, and survival in nonsmall-cell lung cancer patients treated with gefitinib. J Clin Oncol 
DOI: $10.2169 /$ internalmedicine.46.0335

24: 2549-2556, 2006.

17. Hotta K, Kiura K, Tabata M, et al. Interstitial lung disease in Japanese patients with non-small cell lung cancer receiving gefitinib: an analysis of risk factors and treatment outcomes in Okayama Lung Cancer Study Group. Cancer J 11: 417-424, 2005. 18. Fujita J, Ueda Y, Bandoh S, Namihira H, Ishii T, Takahara J. A case of leptomeningeal metastasis from lung adenocarcinoma diagnosed by reverse transcriptase-polymerase chain reaction for carcinoembryonic antigen. Lung Cancer 22: 153-156, 1998.

19. Wasserstrom WR, Glass JP, Posner JB. Diagnosis and treatment of leptomeningeal metastases from solid tumors: experience with 90 patients. Cancer 49: 759-772, 1982.

(C) 2007 The Japanese Society of Internal Medicine http://www.naika.or.jp/imindex.html 